\title{
Coping with the COVID-19 Pandemic's Unique Challenges Associated with Medical Research: A Saudi Institution Approach
}

This article was published in the following Dove Press journal: Risk Management and Healthcare Policy

\author{
Amani Almeharish (D) \\ Abdullah M Assiri ${ }^{2}$ \\ Areej Alfattani $\mathbb{D}^{\prime}$ \\ Edward De Vol (iD)
}

'Department of Biostatics, Epidemiology and Scientific Computing, King Faisal Specialist Hospital and Research Center, Riyadh, Saudi Arabia; ${ }^{2}$ Department of Comparative Medicine, King Faisal Specialist Hospital and Research Center, Riyadh, Saudi Arabia
Correspondence: Amani Almeharish Department of Biostatistics, Epidemiology and Scientific Computing, King Faisal Specialist Hospital and Research Center, P.O. Box 3354, Riyadh I I I I, Kingdom of Saudi Arabia

Email aalmeharish26@kfshrc.edu.sa

\begin{abstract}
The spread of COVID-19 has become a significant threat to economic activity throughout the world, and it has made life particularly difficult for research institutions. According to a report published by Spain's largest public research body, Cybermetrics Labs, King Faisal Specialist Hospital and Research Centre (KFSH\&RC) is ranked first among the leading hospitals in the Arab world, and it has instituted a wide range of policy changes to accommodate researchers by providing them with additional flexibility. The aim of this project was to highlight the most prevalent COVID-related global challenges facing research institutions and describe how research protocol at KFSH\&RC has been adjusted to minimize the disruption experienced by its researchers.
\end{abstract}

Keywords: SARS-CoV-2, COVID-19, healthcare, disease, research, virus

\section{Introduction}

The spread of coronavirus disease 2019 (COVID-19) continues to have a profound impact around the globe, ${ }^{1}$ making it difficult to balance personal safety and work priorities. The World Health Organization (WHO) has recommended several major cities and towns at risk implement lockdown measures and other restrictions to reduce the spread of infection. ${ }^{3,4}$

Most research scientists have faced numerous unexpected difficulties during this period, ${ }^{3,5}$ which is largely due to the lack of access to resources and complications related to the management of personal safety and family responsibilities. ${ }^{6}$ Earlycareer scientists have been impacted the most as they are constantly judged by their research productivity. This is used to promote and secure grant funding. ${ }^{7}$ COVID19 has been particularly challenging for research institutions, though some have benefitted from COVID-related research opportunities. ${ }^{7}$ To deal with the issue of social distancing and lockdowns, many researchers have been forced to work from home. Consequently, many research teams are now spaced too far apart, which complicates collaboration among colleagues. The disruption of ongoing research projects in many fields has also resulted in delayed graduate students data acquisition and analysis. Career opportunities have decreased significantly due to hiring freezes and reduced work schedules, which will lead to increased competition when the pandemic begins to ease. ${ }^{8}$

A substantial amount of institutional research is experiencing some degree of slower growth because most of it is funded by various government entities located 
in developed countries, such as the United States, which has become the global epicenter of the pandemic. Given the level of uncertainty and degradation of the global economy, most of these funds are not being distributed. This has emerged as a serious threat to the continued operations of affected research institutions. ${ }^{9}$

With COVID-19 having such a profound impact on research, most continuous activities, such as long-term laboratory experiments, have been greatly affected. Supplies and equipment procurement is at risk of being postponed or eliminated due to COVID-19. ${ }^{4}$ Many agreements will likely be deferred or terminated because of force majeure circumstances, making it impossible for contracted workers and providers to fulfill their commitments because of ancillary closings or compulsory disconnection.

In response, extraordinary measures have been implemented to reduce transmission of the virus, and many research institutions have continued to operate via remote telework. Working from home can be challenging for many researchers as it requires important equipment and infrastructure (eg, power, internet access, computers, and technology) to be effective. Moreover, lab research involving chemical and biological experiments require physical access to unique facilities and equipment. Collaborating with fellow researchers in the same lab is frequently used to obtain significantly more valuable study results and findings. Virtual lab meetings are not an ideal substitute for spontaneous in-person interactions that generate new ideas and intellectual exploration. It has also become more challenging for professors at institutions to balance their teaching activities and research endeavors.

Extensive restrictions have been placed on travel and personal contact, which has forced research institutions to turn toward online learning, especially for those involved in thesis projects. ${ }^{10}$ Hundreds of global scientific conferences and seminars have also been cancelled or postponed. ${ }^{4}$ While many conferences have shifted to an online format, this presents its own challenges as they often conflict with participants' other personal and professional responsibilities.

Notably, since the initial spread of COVID-19 in early 2020, most of the scientific research institutions have concentrated their efforts on COVID-19-related research and studies. ${ }^{11}$ For instance, the effect of COVID-19 on cognitive processing and mental health has become an important focus. ${ }^{12}$ Moreover, to develop vaccines, effective treatment, and/or rapid testing, most of these institutions paused or canceled other types of scientific research. However, properly designed experiments typically take a significant amount of time to complete ${ }^{8}$ (Inouye, 2020), and thus, the collection of useful research data has become more challenging.

Generally, COVID-19 has resulted in many ineffective and unsustainable research projects. In addition, researchers have been forced to reprioritize experiments and resist the temptation to concentrate solely on COVID-19-related research. The restrictions placed on access to laboratories and completion of field work have led to decreased funding throughout the entire research community.

Many research scientists have sought to work effectively from home by focusing on certain types of work, for example, information investigation, writing audits, composing original copy, proposals, and progress reports. Most inperson gatherings, including diary clubs, are now being conducted remotely. Reviewing plans with every individual from a research group is an option that can facilitate a seminormal remote process, whereby week-by-week plans and progress of those working remotely can be tracked.

\section{Managing Medical Research in a COVID- I 9 Context}

Two types of research have suffered the most due to COVID-19-related considerations, namely animal-based research studies and clinical trials. Animal-based research is particularly challenging. Researchers have been tasked with rapidly arranging investigations that utilize animal models, such as mice, rhesus macaques, and ferrets, to conduct COVID-19 research. In many instances, laboratory animals have become burdensome because scientists are compelled to avoid their laboratories to slow the spread of the infection. Some research scientists have been forced to euthanize their animals, while others have had to halt delicate tests, which will likely require starting over from the beginning with another group of animals after working conditions return to normal. A large number of creatures are utilized in research work in the United States every year. Their lives, similar to our own, have also been disrupted by the pandemic. An ongoing letter to Nature noticed that each lab is "required to have a consideration plan set up for research center creatures in case of a fiasco". 9 The COVID-19 pandemic may speak to that precise situation as it notes that plans must be in place to avoid significant deficiencies in staffing and supply chains. 8,13 
In addition to animal-based research, the continuation of clinical trials has also been problematic. Many institutions, individual principal investigators (PIs), and sponsors have begun using contingency planning to address the potential impact of COVID-19 crisis on current, ongoing clinical trials. This planning includes assessing the value risks associated with continuing the trial as designed or with necessary modifications. Alternatives may include continuing the trial in its present form, conducting the trial in a modified form, as well as suspending or closing the trial. Participation in the trial in accordance with protocol requirements and consideration of alternative models must be conducted to ensure the integrity of the trial is not compromised. Capacity considerations are also important, as the available resources needed to continue the trial must be assessed, including research staff, clinical support staff, pharmacy support, other support staff, space, equipment, and supplies. An additional component of many capacity assessments includes consideration of the need to reallocate research staff to clinical care and other areas of patient support. Contingency planning has become an evolving and ongoing process.

Aside from specific issues related to animal-based research and clinical trials, researchers are generally rethinking their plans for research as a result of the pandemic. Large planned multi-year research programs are being suspended or possibly segmented into smaller shortterm projects. Often times, the experimental execution of smaller short-term studies can be carried out in "bursts" of intense activity with small numbers of participants, thereby accommodating the restrictions associated with the pandemic. Such projects often have budgets that make it possible to overcome the COVID-19-related economic pressures that have constrained research funding (especially non-pandemic research funding). The downside of these changes in research plans will certainly have an impact on overall research productivity, including a loss of continuity often associated with larger, long-term research programs.

In many instances, researchers who practice in fields related to COVID have tended to change their scope of research during this period of time, which has been especially true among ICU physicians, respiratory therapists, epidemiologists, virologists, and genetics researchers. Since it is a new virus, significant efforts have been made to help communities better understand the pandemic through research.
By contrast, researchers who work in fields unrelated to COVID are more likely to be negatively affected by a variety of factors, including delays in processing regulatory forms, the recruitment of participants, a lack of staffing, and/or concerns regarding grant suspension or termination. However, in most instances, such delays would not necessarily result in termination or postponement of the project, rather it causes a wide range of inconvenience and frustration. Logistical considerations aside, a practical solution to resolve delays is to extend the ethical approval for the next 12-18 months while treatments and vaccines are developed and employed, though, it is important to note that this can put the researcher in a precarious situation, especially when the study is funded by a sponsor.

\section{A Saudi Institutional Approach: Mitigating Risk with a Comprehensive Plan}

In 2020, Saudi Arabia's KFSH\&RC was ranked first on the list of leading hospitals in the Arab world according to Spain's largest public research body, Cybermetrics Lab, a research group under the Consejo Superior de Investigaciones Científicas (CSIC). ${ }^{2} \mathrm{KFSH} \& \mathrm{RC}$ offers tertiary and highly specialized inpatient and outpatient medical care, along with active participation in clinical and research studies. Facility management developed and implemented several programs and protocol to address the research issues related to COVID-19 pandemic.

For instance, to address the exposure risks associated with obtaining consent from clinical trial participants, KFH simply stopped recruiting new patients during the pandemic lockdown. Patient recruitment resumed once COVID-19 risks in the hospital clinics decreased to acceptable levels. Even then, many researchers still chose to explain the study to participants by phone in order to obtain the proper consent to be included in the study.

In view of the COVID-19 pandemic, and since $\mathrm{KFSH} \& \mathrm{RC}$ is one of the entities that has been exempted from curfew restrictions, its operations have remained consistent with the Saudi government's Business Continuity Plan. To provide a safe work environment and as part of implementing precautionary measures, all KFSH\&RC employees, managers, and supervisors were required to maintain their work plans, including telework arrangements. Employees working remotely were required to continue their telework arrangements unless otherwise asked by their managers or supervisors. 
KFSH\&RC has addressed other COVID-19 challenges, including a wide variety of policy changes to provide researchers with additional flexibility (Figure 1). First, all staff (including administrative), students, and trainees not directly involved in research lab duties are expected to enter into an electronic contact with their immediate supervisors, allowing them to work remotely. In addition, use of remote technology (learning experience teamwork) has been encouraged, as there are benefits in being able to communicate with investigators around the world to gain a better understand of the policies that have been employed to deal with the pandemic. Some recommendations from investigators have been implemented effectively. For instance, multiple laboratories decided not to accept new studies and animals used for experiments were reduced, while a minimum number of breeding animals have been kept alive.

Other experimental work has also been postponed, and research laboratory work has been limited to maintenance of cell cultures or similar activities. Most ongoing clinical trials were also placed on hold, and clinical trials were instead focused on COVID-19 research. Finally, working hours were shifted for the staff to maintain social distancing, and all training programs were suspended in favor or sharing experiences through webinars and virtual educational courses.

Since this is a global pandemic, and it is affecting people without discrimination, there has been an enormous amount of openness to share information, and many researchers are teaming up rather than competing as they try to find solutions, vaccines, medicine, and so on. KFSH\&RC has a valuable initiative to enhance research collaboration. A COVID-19 workgroup for patients' data has been formed to provide researchers with clinical, diagnostic, and follow-up data for COVID-19 patients through database creation and use. The initiative is a result of teamwork by ICU specialists, immunology scientists, research coordinators, and data managers.

A scientific research taskforce was established to support and accelerate ethics approval of research on the COVID-19 virus. An expedited review process was made available for research relating to COVID-19 or where there are public health grounds for rapid review. In addition, the existence of proper infrastructure led to successful collaborations between KFSH\&RC researchers and others from local universities.

Many factors have contributed to healthcare workers' emotional distress during the pandemic, such as fear of transmitting the virus to fellow healthcare workers, ${ }^{14}$ fear of infecting family members due to workplace exposure, the pressure of making emotionally and ethically fraught resource-allocation decisions, and work-related burnout. ${ }^{15}$ In response, KFSH\&RC launched the employee "Mental Health support Program for COVID-19" to provide mental health support to the employees for any personal help or any concerns about a coworker.

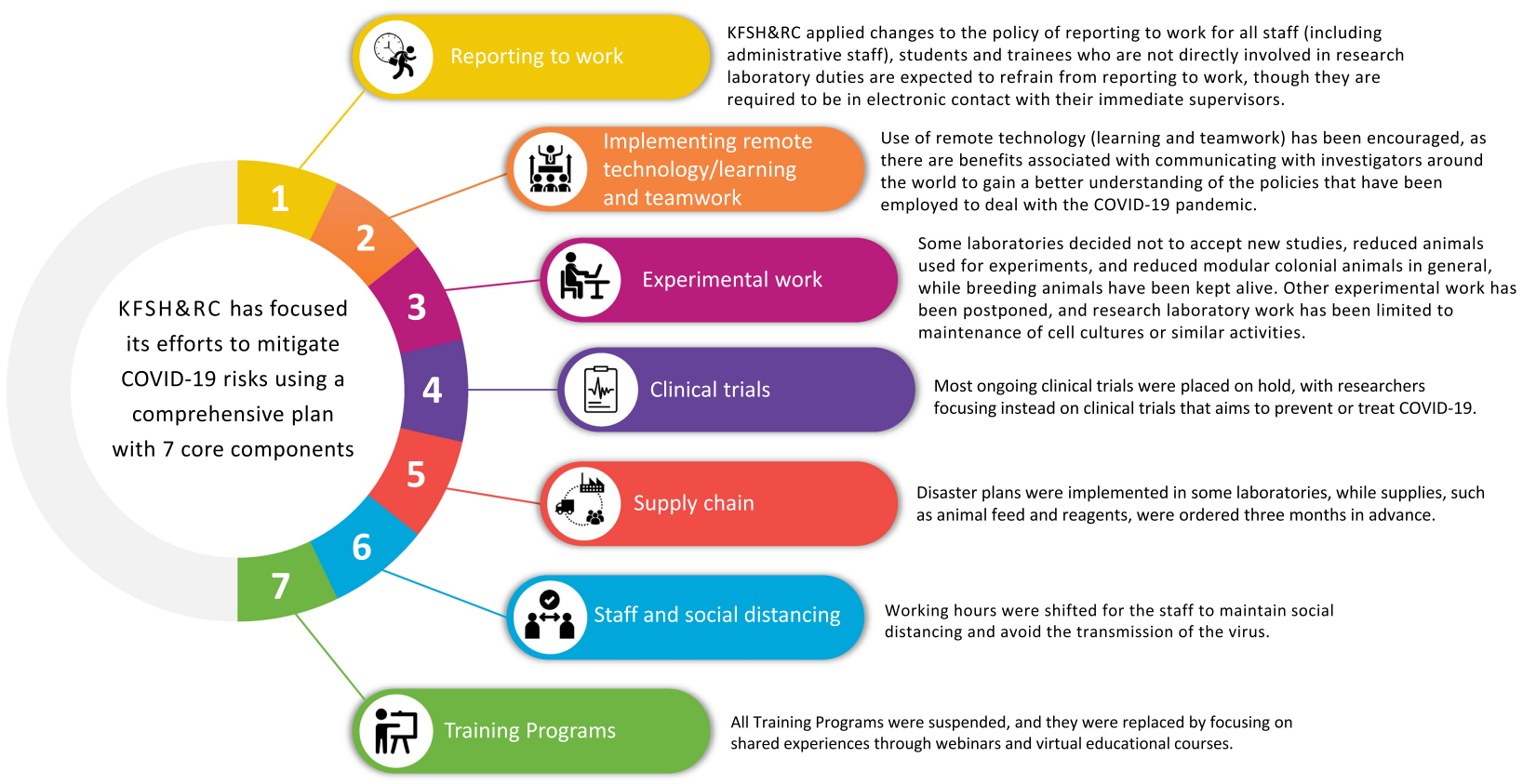

Figure I The COVID-19 challenges addressed by KFSH\&RC.

Note: As this does not meet the scope of a Review, we would like to change the article type to Perspectives. 
While the programs and protocol implemented by KFSH\&RC were largely successful, certain hurdles were not entirely overcome. First, many research projects experienced significant disruptions, and thus, the effectiveness of the facility's strategic plan implementation is somewhat relative. Secondly, while the institution survived and continues to operate, many research projects had to be halted. Finally, logistical complications also affected staffing and created significant disruptions in parts of the supply chain. Disaster plans were implemented in some labs, and animal feed and supplies were ordered three months in advance, though stocking and tracking inventory proved challenging.

Due to the pandemic's adverse economic impact, several studies were affected because a sizable portion of the hospital's budget, particularly research funds, were reallocated to cover patient care expenses. These expenses included personal protective equipment (PPE) and overtime pay for nurses. This trend is likely to continue in the short and intermediate terms. Over the longer term, given that Saudi Arabia has one of the top-20 economies in the world, the country's economic conditions may deteriorate if the spread of the COVID-19 virus cannot be slowed. Moreover, these risk may persist until vaccines and other effective treatment methods are able to control infection and death rates. In the meantime, investment in research infrastructure and local manufacturing of supplies related to COVID-19 has the potential to mitigate the risk of supply chain disruptions and accelerate research findings to combat this and future pandemics.

\section{Conclusions}

COVID-19 has created many challenges for research hospitals and institutions. Facilities have adopted strict protocol, some of which limits and even prevents certain types of research from being conducted, such as experimental lab work and clinical trials. However, as of the writing of this article, KFSH\&RC is COVID-19-free, and mandatory COVID-19 testing is only required for the facility's healthcare workers who work directly with patients, which suggests the program's benefits have outweighed its shortcomings.

\section{Ethical Consideration}

This project received exemption on October 12, 2020, as no vertebrate animals or human subjects were involved or identified by the Institutional Review Board (IRB) of King Faisal Specialty Hospital \& Research Center (KFSH\&RC), Saudi Arabia.

\section{Author Contributions}

Amani Almeharish conceptualized the study and design, drafted the manuscript. Abdullah Assiri and Areej Alfattani participated in the manuscript content. Edward De Vol critically reviewed the manuscript. All authors read and approved the final manuscript. All authors made substantial contributions to conception and design, acquisition of data, or analysis and interpretation of data; took part in drafting the article or revising it critically for important intellectual content; agreed to submit to the current journal; gave final approval of the version to be published; and agree to be accountable for all aspects of the work.

\section{Funding}

There is no funding to report.

\section{Disclosure}

The authors declare that they have no competing interest.

\section{References}

1. Straiton J. COVID-19: how has the scientific community risen to the challenge? BioTechniques. 2020;68(5):232-234. doi:10.2144/btn2020-0041

2. Ranking Web of World Hospitals. (n.d.). Cybermetrics Lab, Consejo Superior de Investigaciones Cientificas (CSIC). Available from: https://hospitals.webometrics.info/en/aw. Accessed July 9, 2020

3. Ghebreyesus TA. Safeguard research in the time of COVID-19. Nat Med. 2020;26:443

4. Nassisi M, Audo I, Zeitz C, et al. Impact of the COVID-19 lockdown on basic science research in ophthalmology: the experience of a highly specialized research facility in France. Eye. 2020;34 (7):1187-1188. doi:10.1038/s41433-020-0944-7

5. Omary M, Eswaraka J, Kimball S, Moghe P, Panettieri R, Scotto K. The COVID-19 pandemic and research shutdown: staying safe and productive. J Clin Invest. 2020;130(6):2745-2748. doi:10.1172/ JCI138646

6. Barnes M, Sax P. Challenges of "Return to Work" in an ongoing pandemic. $N$ Engl J Med. 2020;383(8):779-786. doi:10.1056/ NEJMsr2019953

7. Pain E. How early-career scientists are coping with COVID-19 challenges and fears. Science. 2020. doi:10.1126/science.caredit. abc 3177

8. Inouye DW, Underwood N, Inouye BD, Irwin RE. Support early-career field researchers. Science. 2020;368(6492):724-725.

9. Reidpath D, Allotey P. Preserve Global South's research capacity. Science (New York, NY). 2020;368(6492):725. doi:10.1126/science. abc2677

10. Myers K, Tham WY, Yin Y, et al. Quantifying the immediate effects of the COVID-19 pandemic on scientists. SSRN. 2020. doi:10.2139/ ssrn.3608302

11. Pai M. 'Covidisation' of academic research: opportunities and risks. Nat Res Microbiol Community. 2020. Available from: https://nature microbiologycommunity.nature.com/posts/65638-covidisation-ofacademic-research-opportunities-and-risks.

12. Holmes E, O’Connor R, Perry V, et al. Multidisciplinary research priorities for the COVID-19 pandemic: a call for action for mental health science. Lancet Psychiatry. 2020;7(6):547-560. doi:10.1016/ S2215-0366(20)30168-1 
13. Niriella MA, De Silva AP, De Silva HA, De Silva HJ. Clinical research during the COVID-19 pandemic: gastroenterology researchers' perspective. Frontline Gastroenterol. 2020. doi:10.1136/flgastro-2020-101585

14. Chew NW, Lee GK, Tan BY, et al. A multinational, multicentre study on the psychological outcomes and associated physical symptoms amongst healthcare workers during COVID-19 outbreak. Brain Behav Immun. 2020;88:559-565. doi:10.1016/j.bbi.2020.04.049
15. Pfefferbaum B, North CS. Mental health and the Covid-19 pandemic. $N$ Engl J Med. 2020;383(6):510-512. doi:10.1056/ NEJMp2008017

\section{Publish your work in this journal}

Risk Management and Healthcare Policy is an international, peerreviewed, open access journal focusing on all aspects of public health, policy, and preventative measures to promote good health and improve morbidity and mortality in the population. The journal welcomes submitted papers covering original research, basic science, clinical \& epidemiological studies, reviews and evaluations, guidelines, expert opinion and commentary, case reports and extended reports. The manuscript management system is completely online and includes a very quick and fair peer-review system, which is all easy to use. Visit http://www.dovepress.com/testimonials.php to read real quotes from published authors. 